\title{
A New Robust Signal Recognition Approach Based on Holder Cloud Features under Varying SNR Environment
}

\author{
Jingchao Li \\ School of Electronic Information Engineering, Shanghai Dianji University \\ Shanghai, China \\ [e-mail: lijc@sdju.edu.cn] \\ *Corresponding author: Jingchao Li
}

Received June 18, 2015; revised September 8, 2015; accepted September 23, 2015;

published December 31, 2015

\begin{abstract}
The unstable characteristic values of communication signals along with the varying SNR (Signal Noise Ratio) environment make it difficult to identify the modulations of signals. Most of relevant literature revolves around signal recognition under stable SNR, and not applicable for signal recognition at varying SNR. To solve the problem, this research developed a novel communication signal recognition algorithm based on Holder coefficient and cloud theory. In this algorithm, the two-dimensional (2D) Holder coefficient characteristics of communication signals were firstly calculated, and then according to the distribution characteristics of Holder coefficient under varying SNR environment, the digital characteristics of cloud model such as expectation, entropy, and hyper entropy are calculated to constitute the three-dimensional (3D) digital cloud characteristics of Holder coefficient value, which aims to improve the recognition rate of the communication signals. Compared with traditional algorithms, the developed algorithm can describe the signals' features more accurately under varying SNR environment. The results from the numerical simulation show that the developed 3D feature extraction algorithm based on Holder coefficient cloud features performs better anti-noise ability, and the classifier based on interval gray relation theory can achieve a recognition rate up to $84.0 \%$, even when the SNR varies from $-17 \mathrm{~dB}$ to $-12 \mathrm{~dB}$.
\end{abstract}

Keywords: Communication signal recognition, Holder coefficient cloud features, Interval gray relation classifier, Varying SNR environment 


\section{Introduction}

$\mathbf{T}$ he communication environment in modern communication system is changing and complex. Due to the measurement errors of communication devices themselves and the various interferences caused in transmission environment, there exists a certain degree of randomness and fuzziness between the characteristic parameters of the received communication signals and their real values. Therefore, identifying the communication signals in complex electromagnetic environment accurately has become the key point for modern communication system [1-3]. There are a certain number of established literatures studying signal feature extraction. Higher order cumulants based identification method [4], which has good anti-noise performances, transforms the signals to cumulant domain to process based on the principle that the higher order cumulants of white noise equal to zero. However, this method is only limited to the identification of the white noise environment. Compared with others, the signal recognition algorithm based on wavelet transform [5-6] has been applied widely. The process of this identification algorithm is simple, and has good anti-noise performance. But the number of signals' types can be identified is relatively small. Recognition algorithm based on cyclic spectrum [7] also achieves a good recognition effect. However, it takes a large amount of computation to estimate cyclic spectrum, which greatly limits the application of this algorithm in the field of modulation identification. Fractal dimension [8-9] can describe the complexity features of the signals' waveforms well. Due to the different communication signals' modulation features can reflect on the amplitude, frequency, and phase of carrier wave, signals' waveform variation features can be extracted. This theory definitely provides a new idea and method for the modulation recognition of signals. Yet the current study is limited to the theoretical phase, it still needs time to be further studied and then applied in engineering practice. The feature extraction algorithm based on Holder coefficient [10] has a better intra-class aggregation degree and extra-class separation degree. Wang and Shen [11] made use of the characteristic of Holder coefficient to achieve a good recognition classification result of the radar signal. However, when the SNR is low, the overlap interval becomes too large to identify. Cloud model [12], a qualitative and quantitative uncertainty transformation model, combines the fuzziness in the fuzzy set theory with the randomness in probability theory to overall describe the features of cloud cluster through calculating three different types of digital features---Ex (expected value), En (entropy), and He (hyper entropy), which can be applied to further feature extraction.

The main contributions of this research are as follows:

1. Proposed a novel feature extraction approach---Holder cloud characteristics algorithm, which had good anti-noise ability.

2. Realized the accurate signal recognition at varying SNR condition, which is suitable under today's complex electromagnetic environment. 
The remainder of the paper is organized in four sections. In section 2, the system model of recognition algorithm based on Holder coefficient and cloud theory is presented. Section 3 includes a summary of the basics of Holder coefficient and cloud characteristics algorithm, and also proposes the new approach- Holder cloud characteristics algorithm for communication signal recognition. The application steps of this new approach are also defined. Section 4 demonstrates the numerical simulations of Holder coefficient feature extraction under stable SNR and unstable SNR, and then compares them with traditional cyclic spectrum entropy feature extraction algorithm. The simulation results were compared and analyzed in detail. Section 5 concludes with a further discussion of the insights of the developed communication signal recognition approach, as well as pointing out the future work.

\section{Communication signal recognition model}

Communication signal modulation recognition technique [13-15] is extremely significant in the field of signal processing. It identifies the modulation types of the signals in complex noise environment, and thus provides the fundamental data for further analysis and processing. Communication signal modulation recognition technique mainly consists of two steps- feature extraction and classifier design. The innovation of this research is to provide a new feature extraction algorithm based on Holder cloud characteristics. The communication signal recognition system is shown in Fig. 1.

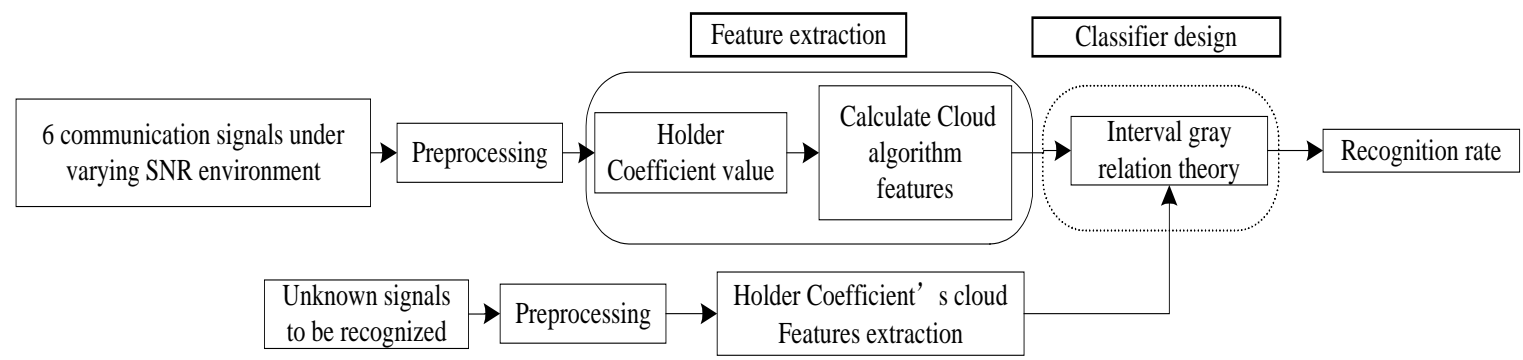

Fig. 1. Communication signal recognition model based on Holder cloud features

In this research, six different communication signals- Amplitude Modulation (AM), Frequency Modulation (FM), Phase Modulation (PM), Amplitude Shift Keying (ASK), Frequency Shift Keying (FSK), Phase Shift Keying (PSK) are taken as samples of communication signals to be recognized. The recognition processes are divided into three main steps. Firstly, the different received communication signals under varying SNR environment are preprocessed to convert the signals from time domain to frequency domain. Secondly, the previous studies[11] suggest to extract Holder coefficient characteristics of signals. This research proposed Holder coefficient cloud features combining Holder coefficient and cloud theory together. Finally, the classifier based on gray relation theory will be employed to recognize the processed communication signal.

Holder coefficient characteristics of communication signals have a certain fuzziness and 
randomness due to the changing noise environment. The distribution characteristics of Holder coefficient are similar with the distribution characteristics of cloud model, which will be further explained in section 3.2. Three digital characteristics, Ex, En, and He of cloud model are used to calculate the Holder coefficient characteristics, in order to better describe the fuzziness and randomness characteristics of the Holder coefficient cloud distribution. In this way, more accurate frequency domain characteristics of signals can be obtained.

The calculated results of Holder cloud characteristics will be taken as the feature database in this research. When the unknown communication signals are received, gray interval relation theory is used to calculate the interval relation degree between the Holder cloud characteristics of these unknown signals and that of the known signals in the database to achieve the purpose of signal identification.

\section{Methodology}

\subsection{Holder coefficient algorithm}

Holder coefficient can be used to measure the similar degree of two sequences. It is evolved from Holder inequality, which is an important formula in the field of mathematics. For any vectors $X \in C^{n}, Y \in C^{n}$, where $X=\left(x_{1}, x_{2}, \cdots, x_{n}\right)^{T}$ and $Y=\left(y_{1}, y_{2}, \cdots, y_{n}\right)^{T}$, Holder inequality [16] can be defined:

$$
\sum_{i=1}^{n}\left|x_{i} \cdot y_{i}\right| \leq\left(\sum_{i=1}^{n}\left|x_{i}\right|^{p}\right)^{1 / p} \cdot\left(\sum_{i=1}^{n}\left|y_{i}\right|^{q}\right)^{1 / q}
$$

where $p, q>1, \frac{1}{p}+\frac{1}{q}=1$.

According to the definition of the Holder inequality in equation (1), Holder coefficient [17] of the two communication signals can be defined as:

$$
H=\frac{\sum s_{1}(i) s_{2}(i)}{\left(\sum s_{1}^{P}(i)\right)^{1 / p} \cdot\left(\sum s_{2}^{q}(i)\right)^{1 / q}}
$$

where $p, q>1, \frac{1}{p}+\frac{1}{q}=1, \quad 0 \leq H \leq 1$, and the value of signals $\left\{s_{1}(i)\right\}, \quad\left\{s_{2}(i)\right\}$ are positive.

$H$ equals to one when $s_{1}^{p}(i)=k s_{2}^{q}(i), i=1,2, \cdots, N, k$ is a real number, which means that the signals belong to the same modulation type. $H$ equals to zero when $\sum s_{1}(i) s_{2}(i)=0$, which means signals belong to completely different modulation types. The value of $H$ lies between 0 and 1 when the two signals have some similar features to some degree. The higher the degree of similarity is, the greater the value of $H$ will be. Due to the advantage of simple calculation and good interclass gathered degree, Holder 
coefficient has been widely used in the field of mathematics analysis and radar signal processing etc. But with the reduction of varying SNR, its characteristics are not stable, which are difficult to identify. So when under the varying SNR environment, cloud model theory was used to extract the Holder coefficient distribution characteristics in this research, in order to improve the recognition results.

\subsection{Cloud characteristics algorithm}

Cloud model, which is the result of advancements in quantum mechanics, put forward by Werner Heisenberg in 1925 [18], has been widely applied in nature language processing,data excavation,decision analysis, intelligent control, image processing, and system performance evaluation [19-20], etc. It fully combines the fuzziness (the bigger the fluctuations in the value range of the object, the fuzzier the concept) with randomness (the randomness of the membership degree) of the object to be described, which constitutes a mutual mapping between the qualitative (the concept of theory domain) and the quantitative study (the membership degree of theory domain's attribute value). Due to its advantage that can describe the points' distribution by quantitative values, it provides a strong theoretical basis for the feature extraction of communication signals. But it requires the object to be described must follow normal distribution, which limit the application of cloud model.

The mathematical equation of cloud model $\mu$ can be expressed as [21]:

$$
\mu=\exp \left[-\frac{\left(x_{i}-E x\right)^{2}}{2 E n^{2}}\right]
$$

where $X_{i}$ is arbitrary value of the discussed domain, $E x$ is the center value of the discussed domain, En is the entropy concept of the discussed domain.

The distribution of the points included in cloud model can be illustrated as Fig. $\mathbf{2}$.

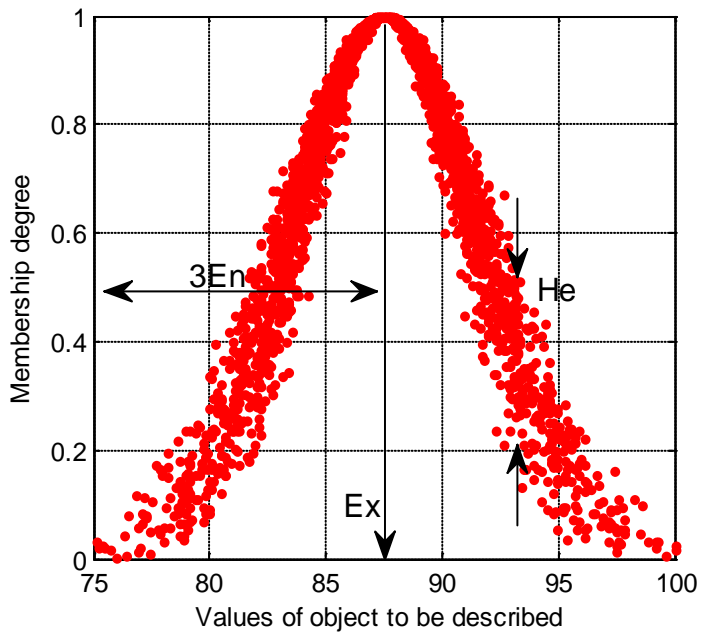

Fig. 2. Distribution of cloud model 
Three digital features- Ex, En, and $\mathrm{He}$ of cloud model are used to characterize an overall concept, and the characteristics of each feature can be described as follows:

(1) Ex (expected value) is the numerical expectation of cloud droplets in the spatial distribution, which can well represent the qualitative concept. It also reflects the center of gravity position of cloud droplets group.

(2) En (entropy) is an uncertainty measurement of qualitative and quantitative concept. On one hand, it is a random measure of qualitative concept, which reflects the discrete degree of cloud droplets that can represent the qualitative concept; on the other hand, it is a fuzzy measure of quantitative concept, which reflects the value scope of cloud droplets that can be accepted in the theory domain.

(3) $\mathrm{He}$ (hyper entropy) reflects the cohesion of uncertainty degree of all the points in the theory domain, i.e. cloud droplets' cohesion. It is an uncertainty measurement of entropy, and its size indirectly expresses the discrete degree and thickness of the cloud, which is decided by combining the randomness and fuzziness of entropy together.

From the definition of cloud model, the points of cloud model follow normal distribution. The Holder coefficient characteristic value of the same communication signal should be the same if there is no noise. When the white noise is added to the signals, the signal waveform will change randomly. At the moment, the Holder coefficient value will fluctuate around a fixed value. When the features are extracted under varying SNR environment, the fluctuation will increase. Therefore, the distributions of characteristic values are around a fixed value which complies with normal distribution. Thus we propose to apply cloud model theory to the feature extraction of communication signals. The digital characteristics of cloud model---Ex, En, and He can be used to reflect the Holder coefficient cloud characteristics under varying SNR environment. Thus, more parameter characteristics in details of different communication signals are extracted under varying SNR environment to identify the signals accurately.

\subsection{Developed Holder cloud characteristics algorithm}

In this research, we innovatively combine Holder coefficient algorithm with cloud model to solve the problem of low signal recognition rate, as well as the existing ambiguity of characteristics under varying SNR environment through twice feature extraction (Holder coefficient characteristics extraction and cloud characteristics extraction). Thus the purpose of accurately recognizing communication signals in lower varying SNR environment can be achieved. The implementation steps of the developed algorithm are demonstrated below:

(1) Under varying SNR environment, six different modulation types of communication signals are preprocessed. The signals are discretized and transformed from time domain to frequency domain.

(2) Suppose the processed signals are:

$$
\left\{S_{n}\left(f_{k}\right), n=1,2, \cdots, 6, k=1,2, \cdots, N\right\}
$$

$n$ is the number of communication signals to be identified. In this research, $n=6$. $N$ is the number of discrete signal points in frequency domain. Then Holder coefficient 
characteristic value of the processed signals to be identified can be calculated. According to the definition of Holder coefficient, the rectangular window function and triangular window function were taken as reference sequences, and they can be set as:

$$
\left\{S_{m}\left(f_{k}\right), m=1,2 ; k=1,2, \cdots, N\right\}
$$

where

$$
S_{1}\left(f_{k}\right)=\left\{\begin{array}{l}
1 \square 1 \leq f_{k} \leq N \\
0, \text { else }
\end{array}\right.
$$

is rectangular window sequence, and

$$
S_{2}\left(f_{k}\right)=\left\{\begin{array}{l}
2 f / N \quad \square 1 \leq f_{k} \leq N / 2 \\
2-2 f / N, N / 2 \leq f_{k} \leq N
\end{array}\right.
$$

is triangular window sequence.

The Holder coefficient value of reference sequence with the signals to be identified can be calculated respectively:

$$
H_{m n}=\frac{\sum_{f_{k}=1}^{N} S_{n}\left(f_{k}\right) S_{m}\left(f_{k}\right)}{\left(\sum_{f_{k}=1}^{N} S_{n}{ }^{p}\left(f_{k}\right)\right)^{1 / p} \cdot\left(\sum_{f_{k}=1}^{N} S_{m}{ }^{q}\left(f_{k}\right)\right)^{1 / q}}
$$

where $m=1,2$, which represents different reference sequences; $n=1,2, \cdots, 6$ which represents the six types of the communication signals; matrix $H_{m n}$ is the extracted Holder coefficient characteristics of the signals.

In addition, the values of $p$ and $q$ directly affect the Holder coefficient characteristics. The distance of Holder coefficient characteristics were calculated through assigning natural numbers to $p$ and $q$ in turn. The value of $p$ and $q$ that produce the maximum separation degree of Holder coefficient characteristic distance were selected. The calculated values for $p$ and $q$ are 2 and 5 respectively.

(3) Due to the low SNR environment and the fact that SNR keeps changing all the time, the extracted Holder coefficient characteristics exist a certain fuzziness and randomness. If the signals are directly classified and recognized, the identification effect will be bad. Given the distribution of extracted characteristics which is similar to the cloud model, the cloud theory was chosen to represent the fluctuation range's randomness and fuzziness of membership degree of Holder coefficient characteristic values. The extracted eigenvalue was further described to make sure that more characteristics of the signals under low SNR would be extracted accurately. Cloud model characteristic value algorithm based on the calculation of the Holder coefficient characteristics can be described as follows:

Firstly, $E x_{n}$ of each signal can be calculated under varying SNR environment: 


$$
E x_{n}=\frac{1}{M} \cdot \sum_{i=1}^{M} H_{1 n}(i)
$$

In order to constitute a Holder coefficient cloud model, sampling must be done many times. A large amount of Holder values should be calculated to compose the Holder cloud. Here $i=1,2, \cdots, M$ represents the calculation times of Holder coefficient value of the same signal, its value is selected according to the accuracy of recognition result we needed. The larger the $M$ value, the more accurate the result is, and the more time the recognition system cost. So the accuracy and the calculation time should be balanced to select the value of $M$. We choose $\mathrm{M}=20$ in this research. $n=1,2, \cdots, 6$ stands for the types of the signals. According to the definition of digital characteristics algorithm of cloud model, the entropy of Holder coefficient characteristics can be calculated as:

$$
E n_{n}(i)=\sqrt{-\frac{\left(H_{1 n}(i)-E x_{n}\right)^{2}}{2 \cdot \ln \left(H_{2 n}(i)\right)}}
$$

Then, the excess entropy of Holder coefficient characteristics can be calculated as:

$$
H e_{n}=\sqrt{\frac{1}{M-1} \sum_{i=1}^{M}\left(E n_{n}(i)-E n_{n}\right)^{2}}
$$

where

$$
E n_{n}=\frac{1}{M} \cdot \sum_{i=1}^{M} E n_{n}(i)
$$

is the average value of the Holder coefficient cloud characteristics entropy.

After calculating three digital characteristics of Holder coefficient characteristics - Ex, En, and He, distribution characteristics of Holder coefficient of communication signals can be expressed clearly under varying SNR environment. Cloud model was used here to represent the randomness and fuzziness characteristics of Holder coefficient distribution. Cloud model overcomes the unstable eigenvalue problems under unstable SNR environment, which helps accomplish the objective of signal identification at low varying SNR.

\section{Simulation results and analysis}

In order to compare the differences of feature extraction under stable SNR and varying SNR environment, numerical simulation under both conditions were made. Then Holder coefficient cloud characteristics of different signals were extracted. Gray interval relation algorithm, which has a good recognition rate for the interval distribution characteristics, was employed to identify the signals. Recognition rates under different varying SNR were calculated. The specific simulation process and results were demonstrated below.

Six different modulation types of communication signals- AM, FM, PM, ASK, FSK, PSK were selected, and suppose the corresponding bandwidth and carrier frequency of the 
six signals were known. The modulation parameters of these six signals were set in Table 1:

Table 1. Modulation parameters of six communication signals

\begin{tabular}{ll}
\hline Signals & \multicolumn{1}{c}{ Parameters' value } \\
\hline AM & $f_{z}=2.7 \times 10^{8} \mathrm{~Hz}, f_{s}=4.32 \times 10^{9} \mathrm{~Hz}, f_{m}=1.0 \times 10^{5} \mathrm{~Hz}, A=1, a=0.8$ \\
FM & $f_{z}=2.7 \times 10^{8} \mathrm{~Hz}, f_{s}=4.32 \times 10^{9} \mathrm{~Hz}, f_{m}=1.0 \times 10^{5} \mathrm{~Hz}, k_{f}=6$ \\
PM & $f_{z}=2.7 \times 10^{8} \mathrm{~Hz}, f_{s}=4.32 \times 10^{9} \mathrm{~Hz}, f_{m}=1.0 \times 10^{5} \mathrm{~Hz}, k_{p}=5$ \\
ASK & $f_{z}=2.7 \times 10^{8} \mathrm{~Hz}, R_{b}=1.0 \times 10^{5} \mathrm{bps}, 0,1$ keying \\
FSK & $f_{z}=2.7 \times 10^{8} \mathrm{~Hz}, R_{b}=1.0 \times 10^{5} \mathrm{bps}, f_{1}=f_{z}-\Delta f, f_{2}=f_{z}+\Delta f, \Delta f=1.0 \times 10^{5} \mathrm{~Hz}$ \\
PSK & $f_{z}=2.7 \times 10^{8} \mathrm{~Hz}, R_{b}=1.0 \times 10^{5} \mathrm{bps}, 0, \pi$ binary phase \\
\hline
\end{tabular}

where, $f_{z}$ is the carrier frequency, $f_{s}$ is the sampling frequency, $f_{m}$ is the baseband signal frequency, $A$ is the amplitude of AM signal, $a$ is the amplitude modulation index, $k_{f}$ is the frequency modulation index, $k_{p}$ is the phase modulation index, $R_{b}$ is the symbol rate, $f_{1}$ and $f_{2}$ are the two frequencies of FSK signal, and $\Delta f$ is the differential frequency.

Before simulating the feature extraction results under varying SNR environment, the simulated results based on Holder coefficient under different but stable SNR environments $(8 \mathrm{~dB}, 2 \mathrm{~dB},-8 \mathrm{~dB},-11 \mathrm{~dB})$ were demonstrated in Fig. 3. The two characteristic values constitute a 2D feature matrix. The abscissa (H1) represents the Holder coefficient characteristic value between the signal to be identified and the reference rectangular window function, while the ordinate (H2) represents the Holder coefficient characteristic value between the signal to be identified and the reference triangle window function.
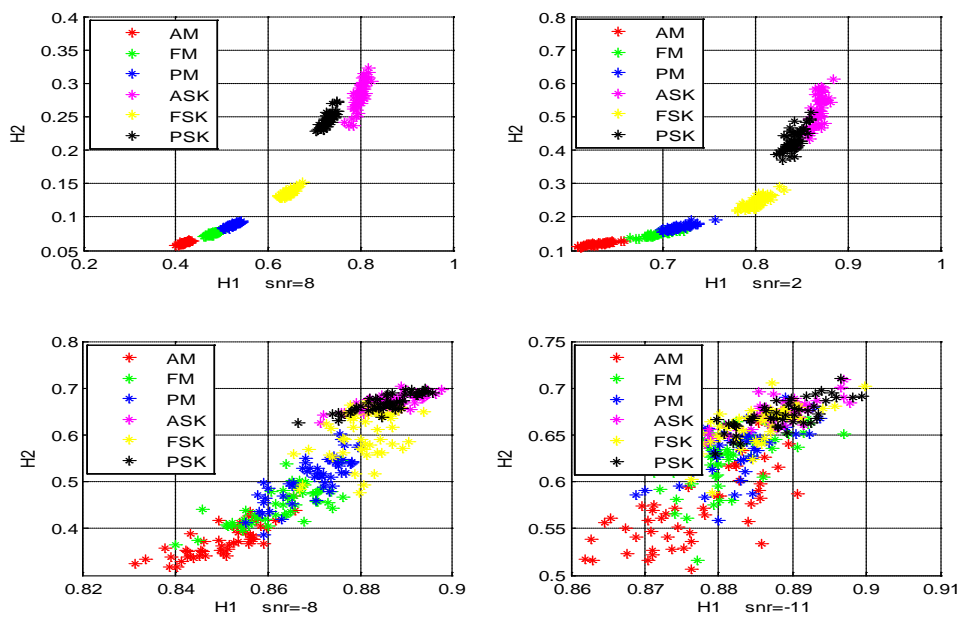

Fig. 3. Holder coefficient characteristics of six communication signals under stable SNR environment 
Unstable noise of the same changing range was added to the signals of different modulation types, and Holder coefficient features of the six communication modulation signals were extracted under different varying SNR environment of $6 \mathrm{~dB} \sim 10 \mathrm{~dB}, 0 \mathrm{~dB} \sim$ $4 d B,-10 d B \sim-6 d B$, and $-15 d B \sim-11 d B$. Simulation results were shown in Fig. 4. Where $H 1$ and $H 2$ have the same meaning with that in Fig. 3.
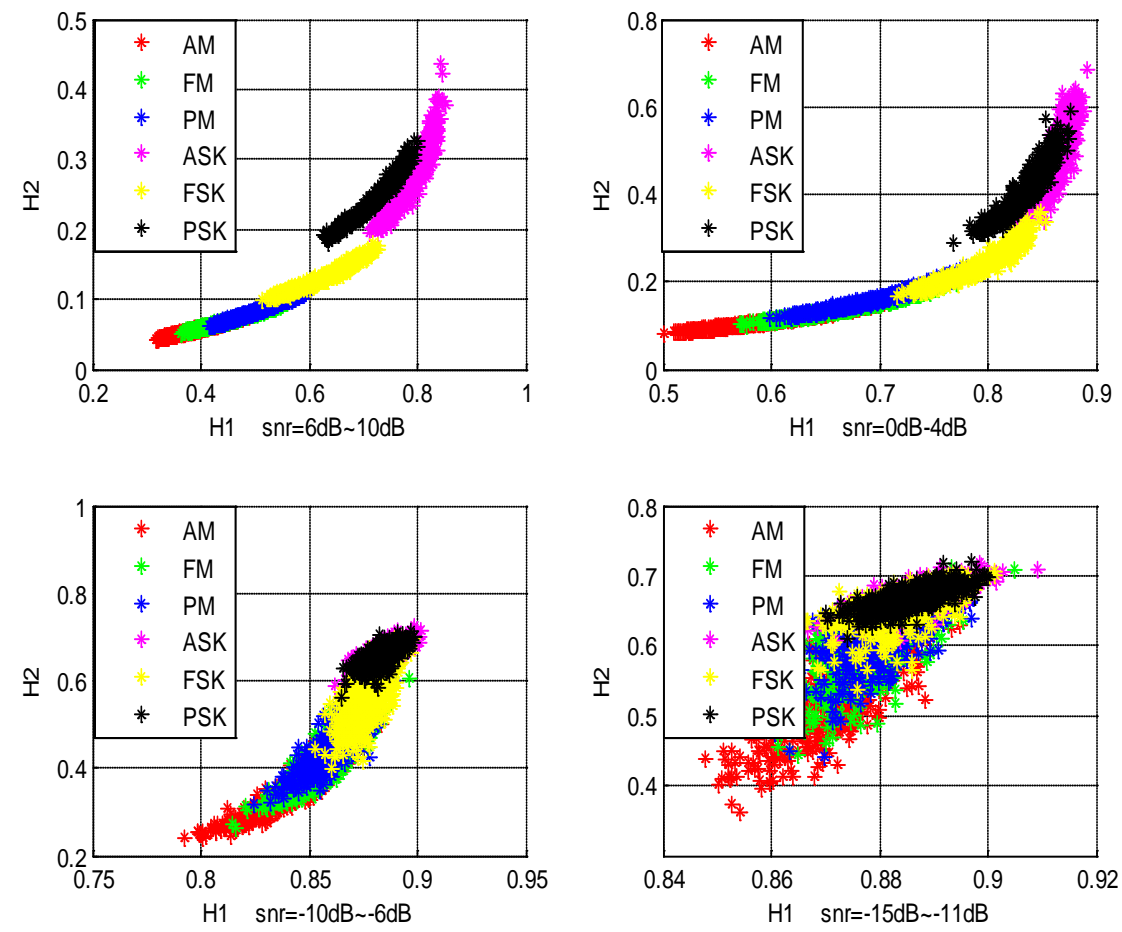

Fig. 4. Holder coefficient characteristics of six communication signals under varying SNR environment

Comparing Fig. 3 with Fig. 4, we found that the features have better aggregation degree under stable SNR environment. However, in today's electromagnetic environment, the noise is becoming more and more complex and the SNR cannot keep stable as assumed in Fig. 3. In practice, the SNR keeps changing all the time within a certain range as we indicated in Fig. 4. Compared with traditional feature extraction methods under a fixed SNR, the topic studied in this research has more application value.

Fig. 4 shows that, under high SNR such as $6 \mathrm{~dB} \sim 10 \mathrm{~dB}, 0 \mathrm{~dB} \sim 4 \mathrm{~dB}$, Holder coefficient characteristics of the signals have good cluster degree. Gray interval relation algorithm can be used to recognize the signals accurately. The overlapping intervals of the six signals increase along with the reduction of SNR gradually. When the SNR reached to -10dB $-6 \mathrm{~dB},-15 \mathrm{~dB} \sim-11 \mathrm{~dB}$, the overlapping interval was too large to achieve high recognition effect even if gray interval relation algorithm was applied. 
Since signal recognition result with Holder coefficient characteristics is not very satisfying, especially when SNR declines, as shown in Fig. 4, we suggest the developed algorithm be applied in feature extraction of the six communication signals. Therefore, Holder cloud characteristics of the signals---a twice feature extraction algorithm were calculated under the varying SNR of $6 \mathrm{~dB} \sim 10 \mathrm{~dB}, 0 \mathrm{~dB} \sim 4 \mathrm{~dB}, 10 \mathrm{~dB} \sim-6 \mathrm{~dB}$, and $-15 \mathrm{~dB} \sim$ $-11 \mathrm{~dB}$. The above four groups of SNR changing ranges stay the same with that in Fig. 4. We performed the simulation and the results were demonstrated in Fig. 5.
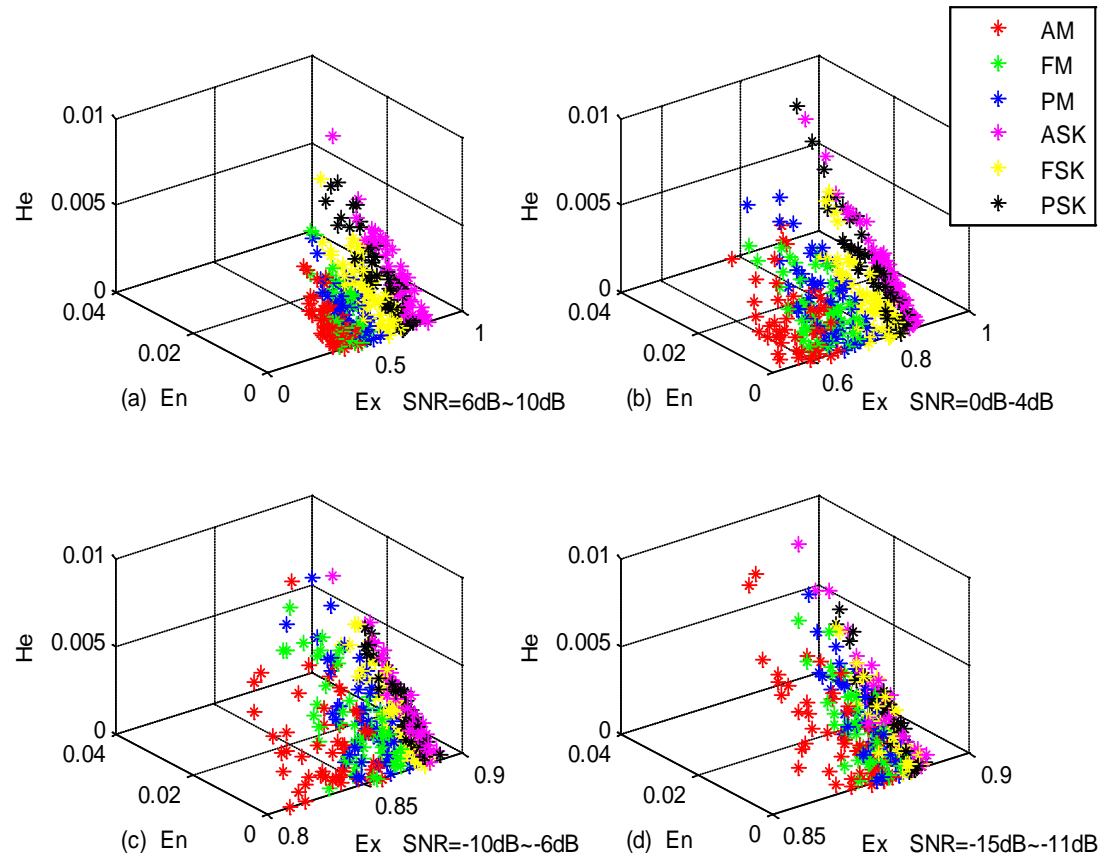

Fig. 5. Holder cloud characteristics of six communication signals under different varying SNR environment

It can be seen that, after twice feature extraction, 2D Holder coefficient characteristics of the signals were transformed to 3D Holder coefficient cloud characteristics. Due to the random distribution property of Holder coefficient features, there is also some instability in $3 \mathrm{D}$ Holder cloud characteristics. Under the higher SNR conditions of $6 \mathrm{~dB} \sim 10 \mathrm{~dB}$ and $0 \mathrm{~dB}$ $\sim 4 \mathrm{~dB}$, the 3D characteristics of the signals have good separation degree; while when the SNR is down to $-10 \mathrm{~dB} \sim-6 \mathrm{~dB},-15 \mathrm{~dB} \sim-11 \mathrm{~dB}$, the overlapping interval is increasing. However, even if overlapping ranges of signal characteristics are increased under lower SNR environment, compared with the Holder coefficient characteristics (Fig. 4), the separating degree is still improved significantly (Fig. 5). Therefore the developed algorithm performs much better in feature extraction of communication signals, which improves the signal recognition rate indirectly. 
After extracting the features of six different communication signals, gray interval relation algorithm based on entropy weighting coefficient [22] was used to identify the characteristics of the signals. During the process of classifying the signals, entropy weighting algorithm was applied for assigning weighting coefficient for each feature automatically. The contribution for classification results of each characteristic can be determined by calculating the separating degree of the characteristics. That being said, the greater weighting coefficient is assigned to the characteristic with greater separating degree, while the smaller weighting coefficient is assigned to the characteristic with smaller separating degree. To achieve a better classification effect, this classification algorithm already performed a very good feature selection before classifying the signals. Compared the recognition rate of traditional signal recognition method based on cyclic spectrum entropy algorithm[23,24] with the feature extraction algorithm based on Holder coefficient theory [25] and Holder cloud characteristics under varying SNR environment, the results were shown in Table 2.

Table 2. Recognition rates of communication signal based on Holder coefficient characteristics and Holder cloud characteristics under different varying SNR environment

\begin{tabular}{ccccccc}
\hline SNR(dB) & $\mathbf{0} \sim 5$ & $\mathbf{- 5} \mathbf{0}$ & $\mathbf{- 1 0} \sim \mathbf{5}$ & $\mathbf{- 1 5}-\mathbf{1 0}$ & $\mathbf{- 1 7}-\mathbf{1 2}$ & $\mathbf{- 2 0 \sim - 1 5}$ \\
\hline Cyclic spectrum entropy features & 65.0 & 60.7 & 50.3 & 47.7 & 46.3 & 35.7 \\
Holder coefficient features (\%) & 100.0 & 98.3 & 94.0 & 65.4 & 44.7 & 28.0 \\
Holder cloud features (\%) & 100.0 & 100.0 & 100.0 & 96.0 & 84.0 & 71.7 \\
\hline
\end{tabular}

In order to see the time complexity of three algorithms, we calculated the simulation time of them, the results were shown in Table 3.

Table 3. Simulation time of three algorithms

\begin{tabular}{llll}
\hline $\begin{array}{l}\text { Name of the } \\
\text { algorithm }\end{array}$ & $\begin{array}{l}\text { Cyclic spectrum entropy } \\
\text { algorithm }\end{array}$ & $\begin{array}{l}\text { Holder coefficient } \\
\text { algorithm }\end{array}$ & $\begin{array}{l}\text { Holder cloud } \\
\text { algorithm }\end{array}$ \\
\hline Time & $22 \mathrm{~ms}$ & $12.5 \mathrm{~ms}$ & $14.6 \mathrm{~ms}$ \\
\hline
\end{tabular}

From Table 2 and Table 3 we can see that, cyclic spectrum entropy algorithm is not suitable for recognition under varying SNR environment. Its anti-noise ability is very poor. Furthermore, it is more complexity than the other two algorithms, because it needs to take a large amount of computation to estimate cyclic spectrum, which greatly limits the application of the algorithm. Holder coefficient algorithm is the simplest, it is more suitable for real time recognition, but with the reduction of varying SNR, it is difficult to recognize the signals accurately. If we want to get the more accurate recognition result under low varying SNR environment, Holder cloud algorithm is the best for recognition.

To get the differences between the cyclic spectrum entropy based, Holder coefficient based and Holder cloud based recognition results of each communication signal, simulations were made and results were shown in Table 4, Table 5 and Table 6. 
Table 4. Recognition rate of each communication signal based on

cyclic spectrum entropy characteristics under different varying SNR environment

\begin{tabular}{ccccccc}
\hline SNR(dB) & $\mathbf{0} \sim 5$ & $\mathbf{- 5 ~ 0}$ & $\mathbf{- 1 0 ~ - 5}$ & $\mathbf{- 1 5}-\mathbf{1 0}$ & $\mathbf{- 1 7}-\mathbf{1 2}$ & $\mathbf{- 2 0 ~ - 1 5}$ \\
\hline AM(\%) & 76.0 & 72.0 & 66.0 & 42.0 & 32.0 & 30.0 \\
FM(\%) & 68.0 & 60.0 & 66.0 & 38.0 & 36.0 & 24.0 \\
PM(\%) & 66.0 & 62.0 & 46.0 & 48.0 & 44.0 & 36.0 \\
ASK(\%) & 58.0 & 56.0 & 50.0 & 52.0 & 62.0 & 34.0 \\
FSK(\%) & 48.0 & 48.0 & 50.0 & 54.0 & 56.0 & 44.0 \\
PSK(\%) & 74.0 & 66.0 & 46.0 & 52.0 & 48.0 & 46.0 \\
\hline
\end{tabular}

Table 5. Recognition rate of each communication signal based on Holder coefficient characteristics under different varying SNR environment

\begin{tabular}{ccccccc}
\hline SNR(dB) & $\mathbf{0} \sim 5$ & $\mathbf{- 5 ~ 0}$ & $\mathbf{- 1 0 ~ - 5}$ & $\mathbf{- 1 5 ~ - 1 0}$ & $\mathbf{- 1 7 ~ - 1 2}$ & $\mathbf{- 2 0 ~ - 1 5}$ \\
\hline AM(\%) & 100.0 & 100.0 & 98.0 & 54.0 & 52.0 & 42.0 \\
FM(\%) & 100.0 & 90.0 & 86.0 & 74.0 & 66.0 & 30.0 \\
PM(\%) & 100.0 & 100.0 & 88.0 & 50.0 & 44.0 & 16.0 \\
ASK(\%) & 100.0 & 100.0 & 86.0 & 66.0 & 62.0 & 48.0 \\
FSK(\%) & 100.0 & 100.0 & 100.0 & 30.0 & 10.0 & 2.0 \\
PSK(\%) & 100.0 & 100.0 & 42.0 & 38.0 & 34.0 & 30.0 \\
\hline
\end{tabular}

Table 6. Recognition rate of each communication signal based on Holder cloud characteristics under different varying SNR environment

\begin{tabular}{ccccccc}
\hline SNR(dB) & $\mathbf{0} \sim 5$ & $\mathbf{- 5 \sim 0}$ & $\mathbf{- 1 0} \sim \mathbf{5}$ & $\mathbf{- 1 5}-\mathbf{1 0}$ & $\mathbf{- 1 7}-\mathbf{- 1 2}$ & $\mathbf{- 2 0 \sim - 1 5}$ \\
\hline AM(\%) & 100 & 100 & 100 & 100 & 100 & 66 \\
FM(\%) & 100 & 100 & 100 & 76 & 62 & 60 \\
PM(\%) & 100 & 100 & 100 & 100 & 58 & 50 \\
ASK(\%) & 100 & 100 & 100 & 100 & 100 & 78 \\
FSK(\%) & 100 & 100 & 100 & 100 & 84 & 76 \\
PSK(\%) & 100 & 100 & 100 & 100 & 100 & 100 \\
\hline
\end{tabular}

Gray interval relation algorithm based on entropy weight has good recognition effect for classifying both the Holder coefficient characteristics and the Holder cloud characteristics of communication signals. Even at the varying SNR of $-5 \mathrm{~dB} \sim 1 \mathrm{~dB}$, it still can reach the recognition rate of $100 \%$. The signal recognition performance of the traditional recognition method based on cyclic spectrum entropy features is too poor to be used under varying SNR environment. However, when the SNR continues to reduce, Holder coefficient features recognition rate declines quickly. For Holder cloud characteristics, the characteristics stay relatively stable due to the further feature extraction of Holder coefficient characteristics. Therefore, the improved algorithm can still maintain the good recognition effect under varying SNR. The developed approach can achieve the recognition rate up to $71.7 \%$ even at the varying SNR condition from $-20 \mathrm{~dB}$ to $-15 \mathrm{~dB}$. 


\section{Conclusion and Discussion}

Decision theory based algorithm and pattern recognition algorithm are the most commonly used methods in the field of communication signal recognition. Pattern recognition algorithm consists of two parts--- feature extraction and classifier design. In software defined radio and cognitive radio system, modulation recognition can complete the identification of the unknown modulation mode, which provides the basis for subsequent signal processing. Therefore, the research on communication signal identification technology is significant and has broad application prospects. Nowadays, the fact that noise in communication environment has become more complex and the SNR is lower and more unstable makes signal recognition more difficult than ever. So, advanced feature extraction algorithm under varying SNR environment is very necessary.

This research proposes a novel feature extraction algorithm for communication signals based on Holder coefficient cloud characteristics. It makes full use of digital characteristics of cloud model to characterize the distribution of the characteristics. Digital characteristics of cloud model were used to describe the unstable Holder coefficient characteristics of communication signals, and the twice feature extraction was applied to achieve the purpose of better recognition. Six types of communication signals were taken as samples to be recognized under varying SNR environment in this research. Holder cloud characteristics of the signals were extracted first, and then the classifier based on gray interval relation theory was employed to classify the extracted features, which turns out a much better recognition effect. The comparing simulation results show that, the improved algorithm had better concentration degree than the traditional methods, even when the SNR was varying from $-17 \mathrm{~dB}$ to $-12 \mathrm{~dB}$, the developed algorithm can still achieve the recognition rate of $84 \%$. Apparently, the newly developed algorithm has better application value in the field of communication signal recognition under today's complex electromagnetic environment.

A number of methods in signal classification need to know some prior knowledge in the area of communication signals. The approach developed in this research also needs a certain prior knowledge of communication signals to constitute a database and classify the signals, which is the potential drawback of this study. So how to realize the blind recognition of the signals is one of the research topics we plan to explore in future.

In Holder cloud based feature extraction algorithm, triangular wave and square wave were chosen as waveform parameters in this research, which have already achieved good recognition results. However, whether we can choose other referenced waveform to achieve better effect remains to be studied in future work.

\section{References}

[1] O. A. Dobre, A. Abdi, Y. Bar-Ness, and W. Su. "A survey of automatic modulation classification techniques: classical approaches and new trends," IET Communications, vol. 1, pp. 137-156, 2007. Article(CrossRefLink)

[2] Wong, ML Dennis, and Asoke K. Nandi. "Automatic digital modulation recognition using artificial neural network and genetic algorithm." Signal Processing, vol. 84, no. 2, pp. 351-365, 
2004. Article(CrossRefLink)

[3] X. Ge, K. Huang, C.-X. Wang, X. Hong, and X. Yang, "Capacity analysis of a multi-cell multi-antenna cooperative cellular network with co-channel interference," IEEE Transactions on Wireless Communications, vol. 10, no. 10, pp. 3298-3309, 2011. Article(CrossRefLink)

[4] Lan-Xun Wang, Yu-Jing Ren. "Recognition of digital modulation signals based on high order cumulants and support vector machines," ISECS International Colloquium on Computing, Communication, Control, and Management, pp. 271-274, 2009. Article(CrossRefLink)

[5] Farooq, O., and S. Datta. "Wavelet based robust sub-band features for phoneme recognition." IEEE Proceedings-Vision, Image and Signal Processing, 2004, 151(3): 187-193. Article(CrossRefLink)

[6] Pan, Hong, and Liang-Zheng Xia. "Efficient object recognition using boundary representation and wavelet neural network." IEEE Transactions on Neural Networks, vol. 19, no. 12, pp. 2132-2149, 2008. Article(CrossRefLink)

[7] Lei Kong, Zhijun Xu, Jinming Wang, Kexiu Pan. "A novel algorithm for jamming recognition in wireless communication," 2013 6th International Congress on Image and Signal Processing (CISP), vol. 3, pp. 1473-1477, 2013. Article(CrossRefLink)

[8] Thierry Blu and Michael Unser. "Wavelets, fractals, and radial basis functions," IEEE Transactions on Signal Processing, vol. 50, no. 3, pp. 543-553, 2002. Article(CrossRefLink)

[9] Lv T. J., Guo S. B., Xiao X. C.. "Fractal characteristics Study of modulation signals," Science in China(Series E), vol. 31, no. 6, pp. 508-513, 2001. (in Chinese). Article(CrossRefLink)

[10] Hambaba, Ahmed, and E. Huff. "Multiresolution error detection on early fatigue cracks in gears," 2000 IEEE on Aerospace Conference Proceedings, vol. 6, pp. 367-372, 2000. Article(CrossRefLink)

[11] Wang H H, Shen X F. "New intra-pulse feature extraction approach of radar emitter signals," Systems Engineering and Electronics, vol. 31, no. 4, pp. 809-811, 2009.

Article(CrossRefLink)

[12] Xiaojuan Han, Yong Yan, Cheng Cheng, et al. "Monitoring of Oxygen Content in the Flue Gas at a Coal-Fired Power Plant Using Cloud Modeling Techniques," IEEE Transactions on Instrumentation and Measurement, vol. 63, no. 4, pp. 953-963, 2014. Article(CrossRefLink)

[13] Nandi, Asoke K., and Elsayed Elsayed Azzouz. "Algorithms for automatic modulation recognition of communication signals." IEEE Transactions on Communications, vol. 46, no. 4, pp. 431-436, 1998. Article(CrossRefLink)

[14] Y. Liu, O. Simeone, A. M. Haimovich and W. Su, "Modulation Classification via Gibbs Sampling Based on a Latent Dirichlet Bayesian Network," IEEE Signal Process. Lett., vol. 21, no. 9, pp. 1135-1139, 2014. Article(CrossRefLink)

[15] Anchalee Puengnim, Nathalie Thomas, Jean-Yves Tourneret, Josep Vidal. "Classification of linear and non-linear modulations using the Baum-Welch algorithm and MCMC methods," Signal Processing, vol. 90, no. 12, pp. 3242-3255, 2010. Article(CrossRefLink)

[16] Holder, O.,"Ueber einen Mittelwertsatz", Nachrichten von der Konigl. Gesellschaft der Wissenschaften under Georg-Augusts - Universitat zu Gottingen, Band (in German). vol. 2, pp. 38-47, 1889. Article(CrossRefLink)

[17] Chiu, Tsenchieh. "Dielectric constant measurement technique for a dielectric strip using a rectangular waveguide." IEEE Transactions on Instrumentation and Measurement, vol. 52, no. 5, pp. 1501-1508, 2003. Article(CrossRefLink)

[18] Werner Heisenberg. "Uber quantentheoretische Umdeutung kinematischer und mechanischer Beziehungen," Zeitschrift fur Physik. vol. 33, no. 1, pp. 879-893, 1925. Article(CrossRefLink)

[19] Tian, Yuan, et al. "Performance evaluation and dynamic optimization of speed scaling on web 
servers in cloud computing." Tsinghua Science and Technology, vol. 18, no. 3, pp. 298-307, 2013. Article(CrossRefLink)

[20] Khazaei, Hamzeh, Jelena Misic, and Vojislav B. Misic. "Performance analysis of cloud computing centers using $\mathrm{m} / \mathrm{g} / \mathrm{m} / \mathrm{m}+\mathrm{r}$ queuing systems." IEEE Transactions on Parallel and Distributed Systems, vol. 23, no. 5, pp. 936-943, 2012. Article(CrossRefLink)

[21] Li, Deyi, Changyu Liu, and Wenyan Gan. "A new cognitive model: cloud model." International Journal of Intelligent Systems, vol. 24, no. 3, pp. 357-375, 2009. Article(CrossRefLink)

[22] Yibing Li, Jingchao Li, Jian Kang. "Classifier Design Algorithms Aimed at Overlapping Characteristics," Information Technology Journal, vol. 11, no. 8, pp. 1091-1096, 2012. Article(CrossRefLink)

[23] S. Sesham, S. Samrat L. "FPGA implementation and performance study of spectrum sensing based on entropy estimation using cyclic features," Computers and Electrical Engineering, vol. 38, no. 6, pp. 1658-1669, 2012. Article(CrossRefLink)

[24] S. Samrat L, S. Srinu , A. Raveendranadh, S. K. Udgata. "Spectrum sensing based on entropy estimation using cyclostationary features for cognitive radio." In Proc. of 2012 4th International Conference on Communication Systems and Networks, pp. 1-6, 2012. Article(CrossRefLink)

[25] Wang Hai-hua, Shen Xiao-feng. "Feature Analysis of Radar Emitter Signals Based on Symmetrical Holder Coefficient," Space Electronic Technology, vol. 3, pp. 55-59, 2010. Article(CrossRefLink)

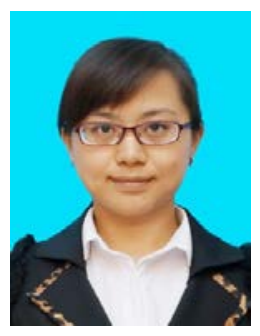

Jingchao Li received the B.S. in Electrical Information Engineering from Harbin Engineering University(HEU) in 2010. During 2010-2011, she stayed in HEU to read M.S.. During 2011-2012, she stayed in HEU to read Ph.D. During 2012-2013, she was in University of Electro-Communication (UEC) of Japan as exchanged student. During 2014.4-2014.6, she studied in Western New England University of USA as a visiting scholar. And in June, 2014, she received the Ph.D. in Electrical Information Engineering from Harbin Engineering University(HEU). Now, she is an instructor of Shanghai Dianji University, China. Her current research interest is communication radiation source recognition, signal feature extraction and classifier design. 